\title{
Dual-buffered SnSe@CNFs as negative electrode with outstanding lithium storage performance
}

Long Zhang ${ }^{\mathrm{a}, *}$, Lei $\mathrm{Lu}^{\mathrm{a}}$, Dechao Zhang ${ }^{\mathrm{a}}$, Wentao $\mathrm{Hu}^{\mathrm{a}}$, Ning Wang ${ }^{\mathrm{a}}, \mathrm{Bo}, \mathrm{Xu}^{\mathrm{a}}$, Yueming $\mathrm{Li}^{\mathrm{a}}$, Hong Zeng

${ }^{a}$ State Key Laboratory of Metastable Materials Science and Technology, Yanshan University, Qinhuangdao, Hebei 066004, China

${ }^{b}$ Beijing Key Laboratory of Energy Nanomaterials, Advance Technology \& Materials Co., Ltd, China Iron \& Steel Research Institute Group, Beijing 100081, P.R. China.

*Corresponding author. Tel.: +86 335 8057047; fax: +86 335 8074545. E-mail address: lzhang@ysu.edu.cn (Long Zhang)

\begin{abstract}
Alloying with Se has been recently found to be an effective way to improve the cycling performance because the reduced-Se formations can act as a buffer matrix against the volume change of electrode for IVA materials or anchors $\mathrm{S}$ in Li-S batteries during cycling. In this paper, SnSe nanoparticles were dispersed within a carbon fiber matrix with ball milling and electrospinning methods. The uniform nano-architecture with $\mathrm{SnSe}$ nanoparticles homogeneously confined in the carbon matrix, which is revealed by X-ray powder diffraction and scanning electron microscope, provides a stable buffer and good electrical conductor for the lithiation/delithiation process. Moreover, Se-based phase acts as an additional stabilizer to alleviate Sn agglomeration during cycling. Such dual-buffered SnSe/C composites thus deliver large reversible capacity of $840 \mathrm{mAh} / \mathrm{g}$ with ultrastable cycling performance over 100 cycles and demonstrate good rate capacity.
\end{abstract}

Keywords: Tin selenide; Lithium-ion batteries; Negative electrode; Electrospinning; Composite nanofibers 


\section{Introduction}

Lithium-ion batteries (LIBs) have not only been widely used in portable electronic devices, but are also now powering hybrid-electric and plug-in electric vehicles and promising to use in stationary energy storage systems. Batteries for these large-scale applications require a significant improvement in the lithium storage capacity $[1,2]$. LIBs have been extensively studied to obtain more efficient storage systems so as to satisfy the growing demands of higher power and energy density for large-scale rechargeable batteries. Since the traditional prevailing graphitized carbon anode materials could not satisfy the increasing demands for high power and high energy because of low theoretical capacity $\left(372 \mathrm{mAh} \mathrm{g}^{-1}\right)$ and poor rate performance [3], Many efforts have been done in the development of new anode materials with high performances and high capacity. Particularly, Sn metal or Sn-based materials have been studied intensively as one of the promising next-generation anode materials for LIBs [4-7], because Sn has a theoretical specific capacity of $993 \mathrm{mAh} \mathrm{g}^{-1}$ if one $\mathrm{Sn}$ atom is fully lithiated by $4.4 \mathrm{Li}$ atoms [8]. However, poor cycling stability and mechanical failure induced by high volume expansion during discharge-charge cycles has prevented their immediate commercialization. In this sense, the strategy that confines well-dispersed nanosized active materials in a carbon matrix have been proved a promising way to improve the structural stability of anode materials, and thus prolonging the cycle life [9-19]. This can be understood based on two aspects. On one hand, nanostructuring is considered to be beneficial to a large specific surface area, a short $\mathrm{Li}^{+}$diffusion length, as well as a mitigated stress generated during cycling, and thus leading to a high $\mathrm{Li}^{+}$diffusion rate and long cycling life. On the other hand, the carbon matrix where nanosized particles finely dispersed acts as a barrier not 
only to avoid the aggregation of nano-particles, but also to serve as an additional buffer to release the stress induced by the volume change of active materials during the lithium alloying-dealloying process. Among a variety of fabrication techniques, each for a different kind of morphology of composite, ball milling and electrospinning are the two commonly used. The former is a simple solution to prepare nanosized powders or mechanical-alloyed compounds [20-22]; the latter is a facile technique to prepare materials with one-dimensional (1D) morphology [23-29]. 1D-hybrid nano-architecture could be favorable for electrochemical performance because of the uniform structure, short ionic-transport lengths, and orientated electron transport along 1D direction [30].

Although the electrochemical performance of Sn composites is strongly affected by the Sn and carbon morphology and their relative spatial configurations, such dependence can be reduced by using $\mathrm{Sn}$ alloy instead of Sn metal and even further weakened if the alloying element with Sn can alloy independently high amount of Li [31]. Recently, alloying with Se (even in a small mole fraction) has been found to be an effective method for enhancing the cycling performance of either $\mathrm{Li}$ - or Na-ion batteries because the reduced-Se formations act as a buffer matrix against the volume change of IVA materials [32-39] or anchors $\mathrm{S}$ in Li-S batteries [40] during cycling. Among $\mathrm{Sn}$-Se compounds, $\mathrm{SnSe}$ is a very interesting semiconductor compound widely used as optical, optoelectronic, and thermoelectric materials. As anode materials for LIBs, $\mathrm{Sn}_{1-n} \mathrm{Se}_{n}$ and $\mathrm{Sn}_{1-n} \mathrm{Se}_{n} / \mathrm{C}$ demonstrates a different electrochemical reaction mechanism with $\mathrm{Li}$ in contrast to $\mathrm{SnO}_{2}$ or $\mathrm{SnS}_{2}$ [38], and show enhanced specific capacity and cycling stability in contrast to metallic Sn though it still encounters the issue of gradual capacity fading $[32,37]$. The specific capacity and cycling 
performance reported in these studies depend on the upper limit of the charging voltage regarding the formation and decomposition of $\mathrm{Li}_{2} \mathrm{Se}\left(\sim 1.5 \mathrm{~V}\right.$ vs. $\left.\mathrm{Li} / \mathrm{Li}^{+}\right)$. If the charging voltage below this voltage value [33, 35], it shows good cycling stability because the uniformly distributed $\mathrm{Li}_{2} \mathrm{Se}$ mitigates the grain growth of $\mathrm{Li}-\mathrm{Sn}$ alloy and $\mathrm{Sn}$ agglomeration. However, the inactive $\mathrm{Li}_{2} \mathrm{Se}$ leads to the irreversible capacity loss. On the contrary, If the charging voltage over this voltage value $[32,37]$, it shows high specific capacity at the cost of cycling stability. Very recently, $\mathrm{SnSe} / \mathrm{C}$ nanocomposite as an anode for Na-ion batteries demonstrate both high reversible capacity and stable cycle performance over 50 cycles [36]. The enhanced cycle performance is attributed to the confinement of Na-Sn domains in the $\mathrm{Na}_{2} \mathrm{Se}$ matrix in addition to the carbon buffer, thus inhibiting $\mathrm{Sn}$ agglomeration. It is therefore addressing the issue here that can both the specific capacity and the cycling performance of SnSe be together optimized via incorporating carbon buffer for LIBs? In this paper, nanosized SnSe prepared by using high-energy ball milling was uniformly incorporated into $1 \mathrm{D}$ carbon nanofibers (CNFs) by using electrospinning technique, as illustrated in Scheme 1. This new 1D nanostructure composites with $\mathrm{SnSe}$ nanoparticles encapsulated in a carbon matrix (SnSe@CNFs) buffer the volume changes during the lithium alloying-dealloying process. Se is activated for the process of Li alloying/de-alloying by charging to a high voltage range (2.5 $\mathrm{V} v s . \mathrm{Li} / \mathrm{Li}^{+}$) to improve the capacity. More important, $\mathrm{Li}_{2} \mathrm{Se}$ formed during cycling serves as a supplementary buffer matrix to alleviate the agglomeration of $\mathrm{Sn}$ particle in $\mathrm{Li}$ intercalation-deintercalation process. SnSe@CNFs therefore demonstrates enhanced electrochemical properties including long cyclability, high capacity, and good Coulombic efficiency. 


\section{Experimental}

\subsection{Synthesis of SnSe@CNFs}

Nanosized SnSe powders were prepared from ball milling commercial SnSe pieces $(99.999 \%$, Alfa). Ball milling was performed in a Fritsch planetary mill (P7 Premium, Fritsch GmbH) with $10 \mathrm{~mm}$ diameter balls for $15 \mathrm{~h}$ at $300 \mathrm{rpm}$. Nanosized SnSe was designated as SnSe-15h. $N, N$-dimethylformamide (DMF, 99.8\%, J\&K Scientific) solutions of Polyacrylonitrile (PAN, $\mathrm{Mw}=150,000$, Aldrich) containing nanosized SnSe powders were strongly stirred at $40{ }^{\circ} \mathrm{C}$ for $20 \mathrm{~h}$. The obtained dispersion was spun into fiber-web via a positively charged syringe nozzle at $15 \mathrm{kV}$ with a flow rate of $0.015 \mathrm{ml} / \mathrm{min}$ and a distance of $12 \mathrm{~cm}$ from the nozzle to the aluminium collector. The electrospun fibers were stabilized at $280{ }^{\circ} \mathrm{C}$ for $1 \mathrm{~h}$ in air and subsequently calcined at $700{ }^{\circ} \mathrm{C}$ and $750{ }^{\circ} \mathrm{C}$, respectively, for $2 \mathrm{~h}$ in $\mathrm{Ar}(95 \mathrm{vol} \%) / \mathrm{H}_{2}(5 \mathrm{vol} \%)$ in a tube furnace to obtain SnSe@ CNF composite nanofibers. Samples cacined at $700{ }^{\circ} \mathrm{C}$ and $750{ }^{\circ} \mathrm{C}$ were designated as $\mathrm{SnSe} @ \mathrm{CNFs}-700$ and $\mathrm{SnSe} @ \mathrm{CNFs}-750$, respectively. For comparison, pure CNFs, denoted CNFs-750, were prepared by using the same electrospinning procedures as SnSe@CNFs-750 just without adding SnSe.

\subsection{Materials characterization}

X-ray powder diffraction (XRD) data were obtained using a Rigaku D/MAX/2500/PC (Cu $\mathrm{K}_{\alpha}$ ). Morphological characterizations of the nanofibers were analyzed with a scanning electron microscope (SEM, Hitachi S-4800 II FESEM) equipped with an energy dispersive spectrometry (EDS) instrument. Transmission electron microscopic (TEM) images and EDS mapping of discharged sample were recorded using a FEI Tecnai G2 F20 field emission TEM equipped with a high-angle annular dark field. Raman scattering measurements were 
performed using a Renishaw inVia system with a $514.5 \mathrm{~nm}$ excitation source. The specific Brunauer-Emmett-Teller (BET) surface area and pore size distribution were measured with Micromeritics ASAP2020 analyzer.

\subsection{Electrochemical measurement}

The anodes were prepared by mixing 70 wt\% active materials (SnSe@CNFs-700, SnSe@CNFs-750, SnSe-15, or CNFs-750), 15 wt\% carbon black (Super-P) and 15 wt\% sodium carboxymethyl cellulose $(\mathrm{CMC})$ binder dissolved in deionized water. The resulting slurry was pasted onto copper foil and dried at $100{ }^{\circ} \mathrm{C}$ for $12 \mathrm{~h}$ under vacuum. The electrochemical properties were investigated using CR2032 coin-type cells assembled in an argon-filled glovebox $\left(\mathrm{H}_{2} \mathrm{O}, \mathrm{O}_{2} \leq 1 \mathrm{ppm}\right)$ using lithium as the counter and reference electrode. The $\mathrm{LiPF}_{6}$ dissolved carbonate-based electrolyte was purchased from Kejingstar LTD. (code No.: LBC305-1) with addition of $5 \%$ fluoroethylene carbonate (FEC). Celgard 2400 was used as a separator film. The galvanostatic charge and discharge measurements were performed on an Arbin BT2000 system. The specific capacities of the composites were calculated based on the total weight of the active materials. Cyclic voltammetry (CV) and electrochemical impedance spectroscopy (EIS) were carried out on a Princeton P4000 electrochemical workstation. The CV measurements were performed in the voltage window of $0.01-2.5 \mathrm{~V}$ versus lithium with a scan rate of $0.1 \mathrm{mV} / \mathrm{s}$ at ambient temperature. The EIS measurements were performed in the frequency range of $0.1 \mathrm{~Hz}-1 \mathrm{MHz}$ at ambient temperature.

\section{Results and discussion}

The XRD patterns of CNFs carbonized at $750{ }^{\circ} \mathrm{C}$ (CNFs-750), nanosized $\mathrm{SnSe}(\mathrm{SnSe}-15 \mathrm{~h})$, 
and carbonized SnSe@CNFs are shown in Fig. 1; the Bragg positions of SnSe diffraction are also indexed accordingly. SnSe-15h demonstrates a dominant $P 4_{2} / n m c$ symmetry, indicating a thermodynamically stable phase of orthorhombic SnSe during long time ball milling. The significant peak broadening of SnSe-15h confirms an effectively reduced grain size via ball milling. After carbonized above $700{ }^{\circ} \mathrm{C}$ (SnSe@CNFs-700 and SnSe@CNFs-750), the SnSe peaks become narrower and sharpened after carbonization due to the growth of agglomerated particles derived from ball milling. This is also seen in the works previously reported [41]. The halo patterns appeared at about $21^{\circ}$ for CNF-contained samples are attributed to the amorphous carbon, which can be clearly seen from CNFs-750.

The SEM images of as-electrospun and carbonized SnSe@CNFs are displayed in Fig. 2a,b and Fig. 2c,d, respectively. The size of SnSe particles mainly locates in the range of several decades nanometers (Fig. 2b), though some of the agglomerated particles with a diameter up to few micrometers are observed (Fig. 2a). However, the big particles outside of the surface of CNFs volatilized after high temperature carbonization. As shown in Fig. $2 \mathrm{c}$ and $\mathrm{d}$, the surface of the fibers contains only nanoparticles with the size less than $100 \mathrm{~nm}$. The EDS elemental mapping images (Fig. 2e) of SnSe@CNFs-750 pellet, cold-pressed from pulverized powders, reveals a homogeneously distribution of $\mathrm{SnSe}$ and carbon. The average atomic percentage ratio of $\mathrm{Se}: \mathrm{Sn}$ is 35.7:36.4, indicating a nearly equal amount of $\mathrm{Sn}$ and Se, i.e., SnSe. This is in accord with the XRD results above mentioned.

Fig. 3a shows a typical CV curve of the SnSe@CNFs-750 electrode at a scan rate of $0.1 \mathrm{mV} / \mathrm{s}$ in the potential range of $0.01-2.5 \mathrm{~V}$. The reduction peaks at $0.28,0.48$, and $0.65 \mathrm{~V}$ during the first cathodic sweep are associated with lithium alloying with $\mathrm{Sn}$ forming a $\mathrm{Li}_{x} \mathrm{Sn}$ alloys, and 
the oxidation peaks at $0.47,0.64,0.73$, and $0.79 \mathrm{~V}$ are assigned to the dealloying reaction of $\mathrm{Li}_{x} \mathrm{Sn}$ alloys. The broad oxidation peaks at $1.16 \mathrm{~V}$ results from lithium extraction from carbon. The oxidation peak at $1.8 \mathrm{~V}$ and the reduction peak at $1.16 \mathrm{~V}$ in the first cycle and shifts toward $1.32 \mathrm{~V}$ in the subsequent cycles can be attributed to the formation and decomposition of SnSe, respectively [38]. The peak shift between the first and subsequent cycles is probably due to the structural change and/or the formation of the solid electrolyte interphase (SEI) layer [36, 38]. Compared with the first cycle, the appearance of the weak peaks at 1.13, 1.44, and $2.11 \mathrm{~V}$ in cathodic sweep in the subsequent cycles we speculate to the formation of polyselenides [42]. It is notable that the CV curves after the first cycle are nearly overlapped, indicating a good electrochemical reversibility of redox reactions with respect to the alloying and dealloying of $\mathrm{Li}$ with $\mathrm{SnSe}$. This is somewhat different from the result previously reported that $\mathrm{Li}_{2} \mathrm{Se}$ formed during discharging is an irreversible conversion because the following charging voltage is below the decomposition voltage of $\mathrm{Li}_{2} \mathrm{Se}\left(\sim 1.5 \mathrm{~V} v s\right.$. $\left.\mathrm{Li} / \mathrm{Li}^{+}\right)$ [33]. Pure SnSe (SnSe-15h) displays a similar CV behavior to SnSe@CNFs, as shown in the inset of Fig. 3a.

The galvanostatic charge/discharge voltage profiles of SnSe@CNFs carbonized at $700{ }^{\circ} \mathrm{C}$ and $750{ }^{\circ} \mathrm{C}$ in the selected cycles between 0.01 and $2.5 \mathrm{~V}$ at the current density of $200 \mathrm{~mA} / \mathrm{g}$ are displayed in upper and lower panels of Fig. 3b, respectively. An initial lithiation capacity of $1148 \mathrm{mAh} / \mathrm{g}$ is achieved for $\mathrm{SnSe} @ \mathrm{CNFs}-750$, where 82\% of the reacted Li can be reversibly delithiated with a charge capacity of $944 \mathrm{mAh} / \mathrm{g}$. The capacity decay of $204 \mathrm{mAh} / \mathrm{g}(18 \%)$ is mainly due to the formation of SEI film by the decomposition of the electrolyte. The initial measured capacity is obviously higher than that of theoretical capacity of $868 \mathrm{mAh} / \mathrm{g}$, with 
the assumption that $\mathrm{Sn}$ fully converts to $\mathrm{Li}_{4.4} \mathrm{Sn}$ based on the reaction expressed by: $\mathrm{SnSe}+$ $6.4 \mathrm{Li} \rightarrow \mathrm{Li}_{4.4} \mathrm{Sn}+\mathrm{Li}_{2} \mathrm{Se}$. The similar cases are also seen in previously studies and can be explained by the origin of the mesoporous structure of the low temperature carbonized carbon $[43,44]$. The SnSe@CNFs-750 composite exhibits a repeatable charge/discharge profiles for the $3^{\text {rd }}, 10^{\text {th }}$, and $100^{\text {th }}$ cycle, indicating a stable cycling performance. The charge and discharge plateaus/shoulders are in the ranges according to the CV results.

Figure 3c shows the cycling performance and Coulombic efficiency of SnSe@CNFs-750 during charge/discharge processes between 0.01 and $2.5 \mathrm{~V}$ at the current density of $200 \mathrm{~mA} / \mathrm{g}$, and compared with those of SnSe@CNFs-700, SnSe-15h, and CNFs-750. With higher carbonized temperature, SnSe@CNFs-750 not only demonstrates an obviously larger specific capacity larger than SnSe@CNFs-700 but also a better cycling performance. The former steadily maintains a reversible capacity of about $840 \mathrm{~mA} / \mathrm{g}$ after the $4^{\text {th }}$ cycle while the capacity of the latter decreases from $830 \mathrm{~mA} / \mathrm{g}$ for the $4^{\text {th }}$ cycle to $730 \mathrm{~mA} / \mathrm{g}$ for the $100^{\text {th }}$ cycle. The two SnSe@CNF composites both show excellent reversibility with a Coulombic efficiency approaching $100 \%$ after few cycles. As an example, the Coulombic efficiency of SnSe@CNFs-750 rapidly increases from $82 \%$ for the $1^{\text {st }}$ cycle to $97 \%$ for the $3^{\text {rd }}$ cycle and stabilizes at $99 \%$ after five cycles, indicating a facile insertion-extraction of $\mathrm{Li}^{+}$processes [45]. The Raman spectra of SnSe@CNTs were measured at ambient condition to clarify the different performance of the two composites carbonized at different temperature, as shown in Fig. 4. The two broad peaks are associated with the $\mathrm{D}$ (around $1350 \mathrm{~cm}^{-1}$ ) and $\mathrm{G}$ (around $1590 \mathrm{~cm}^{-1}$ ) band of carbon materials, which represents the existence of amorphous carbon and graphitic carbon, respectively. The intensity ratio of D/G for SnSe@CNFs-750 $\left(\mathrm{I}_{\mathrm{D}} / \mathrm{I}_{\mathrm{G}}=\right.$ 
0.96) is slightly lower than that for SnSe@CNTs-700 $\left(\mathrm{I}_{\mathrm{D}} / \mathrm{I}_{\mathrm{G}}=0.98\right)$, indicating a higher electrical conductivity with a higher carbonized temperature and thus facilitating the improvement of storage capacity. As expected, pure CNFs (CNFs-750, Fig. 3c) demonstrate a perfect cycling stability. It is worth nothing that although pure SnSe (SnSe-15h, Fig. 3c) shows only a short-term stability up to 30 cycles because of the existence of SnSe large particle and absence of CNF buffer layer, it demonstrates a high specific capacity around 800 $\mathrm{mAh} / \mathrm{g}$ and the cycling performance is much better than that of pure $\mathrm{Sn}$ or $\mathrm{SnO}_{2}$, the capacity of which fast decays just in the first few cycles $[6,45,46]$.

Besides the cycling stability, a good rate capability is favourable for practical application in Li-storage. SnSe@CNFs-750 exhibits excellent rate performance in the current range 100-1800 mA/g, as shown in Fig. 3d. The specific capacity with good retention varies from $860,827,787,730,664,630$, and $590 \mathrm{mAh} / \mathrm{g}$ at 100, 300, 600, 900, 1200, 1500, and 1800 $\mathrm{mA} / \mathrm{g}$, respectively. Moreover, the recoverable capacity is $95 \%$ when the current density turns back to $100 \mathrm{~mA} / \mathrm{g}$. CNFs-750 demonstrates an even better rate performance. At current density higher than $600 \mathrm{~mA} / \mathrm{g}$, the capacities for various current densities are similar, indicating a good electrical conductivity. The recoverable capacity of CNFs-750 is $100 \%$ when the current density turns back to $100 \mathrm{~mA} / \mathrm{g}$. By contrast, although pure $\mathrm{SnSe}$ without CNFs encapsulation ( $\mathrm{SnSe}-15 \mathrm{~h}$ ) shows stable rate cycle performance at the current density below $300 \mathrm{~mA} / \mathrm{g}$, the rate capacity decays rapidly when the current density higher than 600 $\mathrm{mA} / \mathrm{g}$ and cannot be recovered thereafter when the current density turns back to $100 \mathrm{~mA} / \mathrm{g}$. This result is in accord with the behavior of the cycling performance.

The outstanding cycling stability of SnSe@CNFs and superior specific capacity approaching 
theoretical value are believed to be ascribed to the unique buffered structure of $\mathrm{Sn}$ rooted in CNFs, along with the protection of $\mathrm{Li}_{2} \mathrm{Se}$ matrix during cycling. On one hand, from the composite point of view, the CNF matrix not only mitigates the massive volume changes during the lithiation/delithiation process but also enhances the electrical conductivity of the electrode. Moreover, the ball-milled nanoparticles may shorten the $\mathrm{Li}^{+}$diffusion distances. BET and EIS measurements were performed to further understand the effects of CNF matrix. Fig. 5a displays the nitrogen adsorption/desorption isotherm curves and pore-size distribution of CNFs and SnSe@CNFs. A typical IV characteristic and micropore-dominated size distribution are observed. The corresponding specific surface areas are about $428 \mathrm{~m}^{2} \mathrm{~g}^{-1}$ and $124 \mathrm{~m}^{2} \mathrm{~g}^{-1}$, respectively. The significantly decreased BET surface area and reduced size distribution (the inset of Fig. 5a) after SnSe incorporation confirm the impregnation of SnSe in the pore-channels of CNFs. Fig. 5b displays Nyquist plots of the impedance at room temperature for SnSe-15h and SnSe@CNFs-750 before and after cycling test (100 cycles). The impedance spectrum normally represents two distinct features ascribed to the charge transfer resistance on the electrode/electrolyte interface (semicircle at high frequency region) and the mass transfer resistance (long tail at low frequency region). From Fig. 5b, several observations can be made. First, before cycling test, pure SnSe shows a severely depressed shape in comparison with SnSe@CNFs-750, indicating an enhanced conductivity of SnSe@CNFs-750 by incorporating CNFs. Secondly, the diameter of the semicircle and the slope of the long tail for SnSe@CNFs-750 changes slightly after cycling test, indicating a stable charge-transfer resistance and microstructure of the electrode mainly profit from CNFs. On the other hand, $\mathrm{Li}_{2} \mathrm{Se}$ also acts as buffer matrix for $\mathrm{Sn}$ or $\mathrm{Li}-\mathrm{Sn}$ alloy during cycling to 
alleviate the pulverization of $\mathrm{Sn}$ induced by agglomeration, which is also seen in $\mathrm{Sn}_{1-n} \mathrm{Se}_{n}$ anodes $[35,36]$ and Se-anchored $\mathrm{S}_{1-n} \mathrm{Se}_{n}$ cathode [40]. The microstructure and EDS mapping of SnSe@CNFs-750 after the tenth discharges was investigated with TEM (Fig. 6a, b). After full lithiation to $0.01 \mathrm{~V}$, Sn-based nanoparticles dispersed in Se-based matrix as confirmed by the EDS mapping images for Sn and Se (inset of Fig. 6a). It is interesting that the interface (Fig. 6b) between the domains and the matrix exists a transition region with a thin layer of few nanometers thickness (the inset of Fig. 6b). The lithiation/delithiation mechanism of SnSe is further investigated with ex situ XRD at the state after $1^{\text {st }}$ discharged, $100^{\text {th }}$ discharged, and $100^{\text {th }}$ charged, as illustrated in Fig. 6c. Several observations can be made from the XRD profiles. First, $\mathrm{SnSe}$ decomposed and lithiated to $\mathrm{Li}_{x} \mathrm{Sn}$ and $\mathrm{Li}_{2} \mathrm{Se}$ during discharging and recovered to SnSe during charging. Secondly, the XRD diffraction peaks of resulting $\mathrm{Li}_{x} \mathrm{Sn}, \mathrm{Li}_{2} \mathrm{Se}$, and $\mathrm{SnSe}$ are invisible or very weak because these resultants are nearly amorphous phase and/or too small to be identified by XRD technique. Thirdly, small amount of residual Sn is observed, of which XRD intensity decreases with increasing cycles, indicating that most of formed $\mathrm{Sn}$ was lithiated. We speculate that the insolubility of $\mathrm{Li}_{2} \mathrm{Se}$ in carbonate-based electrolytes $[47,48]$ slows down the Li-ion diffusing into $\mathrm{Li}_{2} \mathrm{Se}$-wrapped $\mathrm{Sn}$, and thus resulting in residual $\mathrm{Sn}$. Therefore, in addition to act as a conducting network and buffer layer for Li-Sn alloy, CNFs compose a suitable matrix to confine resulting Se and thus Se directly reducing to insoluble $\mathrm{Li}_{2} \mathrm{Se}$, which provides additional buffer matrix for $\mathrm{Li}-\mathrm{Sn}$ alloy. This dual-buffered system is attributed to the superior electrochemical performance of SnSe@CNFs. 


\section{Conclusions}

SnSe@CNF composites with 1D nanostructures were synthesized using a single nozzle electrospinning method. Ball-milled SnSe nanoparticles were dispersed and confined to carbon nanofibers, providing a stable buffer matrix for $\mathrm{Li}^{+}$alloying/dealloying as well as an enhanced electrical conductivity of the electrode. Moreover, $\mathrm{Li}_{2} \mathrm{Se}$ matrix confined by $\mathrm{CNFs}$ provides a supplementary buffer matrix for alleviating Sn agglomeration during cycling. The SnSe@CNF nanocomposites exhibit a high reversible capacity of $840 \mathrm{mAh} / \mathrm{g}$, approaching the theoretical capacity, without capacity fading over 100 charge/discharge cycles at a rate of $200 \mathrm{~mA} / \mathrm{g}$. In addition, a good rate capability of $590 \mathrm{mAh} / \mathrm{g}$ is still shown with negligible capacity loss at a rate of $1800 \mathrm{~mA} / \mathrm{g}$. Such a strategy of forming multiple-buffer matrix is promising and attractive for developing high-performance electrode materials.

\section{Acknowledgments}

This work was supported by the National Science Foundation of China 51201149 and 51202212, and the Key Basic Research Project of Hebei (14961013D).

\section{References}

[1] Y. Tang, Y. Zhang, W. Li, B. Ma, X. Chen, Rational material design for ultrafast rechargeable lithium-ion batteries, Chem. Soc. Rev. 44 (2015) 5926.

[2] P. Roy, S.K. Srivastava, Nanostructured anode materials for lithium ion batteries, J. Mater. Chem. A 3 (2015) 2454.

[3] N. Nitta, F. Wu, J.T. Lee, G. Yushin, Li-ion battery materials: present and future, Mater. Today, 18 (2015) 252.

[4] A.D.W. Todd, P.P. Ferguson, M.D. Fleischauer, J.R. Dahn, Tin-based materials as negative electrodes for Li-ion batteries: Combinatorial approaches and mechanical methods, Int. J. Energ. Res. 34 (2010) 535. 
[5] B. Wang, B. Luo, X. Li, L. Zhi, The dimensionality of Sn anodes in Li-ion batteries, Mater. Today 15 (2012) 544.

[6] Y. Zhao, X. Li, B. Yan, D. Li, S. Lawes, X. Sun, Significant impact of 2D graphene nanosheets on large volume change tin-based anodes in lithium-ion batteries: A review, J. Power Sources 274 (2015) 869.

[7] M. Wachtler, M. Winter, J.O. Besenhard, Anodic materials for rechargeable Li-batteries, J. Power Sources 105 (2002) 151.

[8] M. Winter, J.O. Besenhard, Electrochemical lithiation of tin and tin-based intermetallics and composites, Electrochim. Acta, 45 (1999) 31.

[9] G. Derrien, J. Hassoun, S. Panero, B. Scrosati, Nanostructured Sn-C composite as an advanced anode material in high-performance lithium-ion batteries, Adv. Mater. 19 (2007) 2336.

[10] D. Deng, J.Y. Lee, Reversible storage of lithium in a rambutan-like tin-carbon electrode, Angew. Chem. Int. Ed. 48 (2009) 1660.

[11] Y. Yu, L. Gu, C. Wang, A. Dhanabalan, P.A. van Aken, J. Maier, Encapsulation of Sn@ carbon nanoparticles in bamboo-like hollow carbon nanofibers as an anode material in lithium-based batteries, Angew. Chem. Int. Ed. 48 (2009) 6485.

[12] Y.G. Yu, Lin; Zhu, Changbao; Aken, Peter A. van; Maier, Joachim, Tin nanoparticles encapsulated in porous multichannel carbon microtubes: Preparation by single-nozzle electrospinning and application as anode material for high-performance Li-based batteries, J. Am. Chem. Soc. 131 (2009) 15984.

[13] I. Meschini, F. Nobili, M. Mancini, R. Marassi, R. Tossici, A. Savoini, M.L. Focarete, F. Croce, High-performance Sn@ carbon nanocomposite anode for lithium batteries, J. Power Sources 226 (2013) 241.

[14] Y. Xu, Q. Liu, Y. Zhu, Y. Liu, A. Langrock, M.R. Zachariah, C. Wang, Uniform nano-Sn/C composite anodes for lithium ion batteries, Nano Lett. 13 (2013) 470.

[15] X. Xia, X. Wang, H. Zhou, X. Niu, L. Xue, X. Zhang, Q. Wei, The effects of electrospinning parameters on coaxial Sn/C nanofibers: Morphology and lithium storage performance, Electrochim. Acta 121 (2014) 345.

[16] H. Qiao, K. Chen, L. Luo, Y. Fei, R. Cui, Q. Wei, Sonochemical synthesis and high lithium storage properties of Sn/CMK-3 nanocomposites, Electrochim. Acta 165 (2015) 149.

[17] J. Wang, W.-L. Song, Z. Wang, L.-Z. Fan, Y. Zhang, Facile fabrication of binder-free metallic tin nanoparticle/carbon nanofiber hybrid electrodes for lithium-ion batteries, Electrochim. Acta 153 (2015) 468.

[18] Y. Yu, Q. Yang, D. Teng, X. Yang, S. Ryu, Reticular Sn nanoparticle-dispersed PAN-based carbon 
nanofibers for anode material in rechargeable lithium-ion batteries, Electrochem. Commun. 12 (2010) 1187.

[19] W.-S. Chang, C.-M. Park, H.-J. Sohn, Electrochemical performance of pyrolyzed polyacrylonitrile (PAN) based Sn/C composite anode for Li-ion batteries, J. Electroanal. Chem. 671 (2012) 67.

[20] L. Su, Y. Jing, Z. Zhou, Li ion battery materials with core-shell nanostructures, Nanoscale 3 (2011) 3967.

[21] C.-M. Park, H.-J. Sohn, A mechano- and electrochemically controlled SnSb/C nanocomposite for rechargeable Li-ion batteries, Electrochim. Acta 54 (2009) 6367.

[22] A. Darwiche, M.T. Sougrati, B. Fraisse, L. Stievano, L. Monconduit, Facile synthesis and long cycle life of $\mathrm{SnSb}$ as negative electrode material for Na-ion batteries, Electrochem. Commun. 32 (2013) 18.

[23] S. Cavaliere, S. Subianto, I. Savych, D.J. Jones, J. Rozière, Electrospinning: designed architectures for energy conversion and storage devices, Energ. Environ. Sci. 4 (2011) 4761.

[24] Z. Dong, S.J. Kennedy, Y. Wu, Electrospinning materials for energy-related applications and devices, J. Power Sources 196 (2011) 4886.

[25] X. Zhang, L. Ji, O. Toprakci, Y. Liang, M. Alcoutlabi, Electrospun nanofiber-based anodes, cathodes, and separators for advanced lithium-ion batteries, Polym. Rev. 51 (2011) 239.

[26] C.L. Zhang, S.H. Yu, Nanoparticles meet electrospinning: Recent advances and future prospects, Chem Soc Rev 43 (2014) 4423.

[27] V. Aravindan, J. Sundaramurthy, P. Suresh Kumar, Y.-S. Lee, S. Ramakrishna, S. Madhavi, Electrospun nanofibers: A prospective electro-active material for constructing high performance Li-ion batteries, Chem. Commun. 51 (2015) 2225.

[28] L. Zeng, X. Wei, J. Wang, Y. Jiang, W. Li, Y. Yu, Flexible one-dimensional carbon-selenium composite nanofibers with superior electrochemical performance for $\mathrm{Li}-\mathrm{Se} / \mathrm{Na}-\mathrm{Se}$ batteries, J. Power Sources 281 (2015) 461.

[29] L. Zeng, W. Zeng, Y. Jiang, X. Wei, W. Li, C. Yang, Y. Zhu, Y. Yu, A flexible porous carbon nanofibers-selenium cathode with superior electrochemical performance for both Li-Se and Na-Se batteries, Adv. Energy Mater. 5 (2015) 1401377.

[30] H.-G. Wang, S. Yuan, D.-L. Ma, X.-B. Zhang, J.-M. Yan, Electrospun materials for lithium and sodium rechargeable batteries: from structure evolution to electrochemical performance, Energ. Environ. Sci. 8 (2015) 1660.

[31] K. Shiva, H.B. Rajendra, A.J. Bhattacharyya, Electrospun SnSb crystalline nanoparticles inside porous 
carbon fibers as a high stability and rate capability anode for rechargeable batteries, ChemPlusChem 80 (2015) 516.

[32] X. Wang, B. Liu, Q. Xiang, Q. Wang, X. Hou, D. Chen, G. Shen, Spray-painted binder-free SnSe electrodes for high-performance energy-storage devices, ChemSusChem 7 (2014) 308.

[33] H.S. Im, Y.R. Lim, Y.J. Cho, J. Park, E.H. Cha, H.S. Kang, Germanium and tin selenide nanocrystals for high-capacity lithium ion batteries: Comparative phase conversion of germanium and tin, J. Phys. Chem. C 118 (2014) 21884.

[34] K.C. Klavetter, J. Pedro de Souza, A. Heller, C.B. Mullins, High tap density microparticles of selenium-doped germanium as a high efficiency, stable cycling lithium-ion battery anode material, J. Mater. Chem. A 3 (2015) 5829.

[35] H.X. Dang, K.C. Klavetter, M.L. Meyerson, A. Heller, C.B. Mullins, Tin microparticles for a lithium ion battery anode with enhanced cycling stability and efficiency derived from Se-doping, J. Mater. Chem. A 3 (2015) 13500.

[36] Y. Kim, Y. Kim, Y. Park, Y.N. Jo, Y.-J. Kim, N.-S. Choi, K.T. Lee, SnSe alloy as a promising anode material for Na-ion batteries, Chem. Commun. 51 (2015) 50.

[37] Z. Zhang, X. Zhao, J. Li, SnSe/carbon nanocomposite synthesized by high energy ball milling as an anode material for sodium-ion and lithium-ion batteries, Electrochim. Acta, 176 (2015) 1296.

[38] M.-Z. Xue, J. Yao, S.-C. Cheng, Z.-W. Fu, Lithium electrochemistry of a novel SnSe thin-film anode, J. Electrochem. Soc. 153 (2006) A270.

[39] J. Choi, J. Jin, I.G. Jung, J.M. Kim, H.J. Kim, S.U. Son, SnSe2 nanoplate-graphene composites as anode materials for lithium ion batteries, Chem. Commun. 47 (2011) 5241.

[40] X. Li, J. Liang, K. Zhang, Z. Hou, W. Zhang, Y. Zhu, Y. Qian, Amorphous S-rich $\mathrm{S}_{1-x} \mathrm{Se}_{x} / \mathrm{C}(x \leq 0.1)$ composites promise better lithium-sulfur batteries in a carbonate-based electrolyte, Environ. Sci. 8 (2015) 3181. [41] A. M;, R. A;, F. M;, B. P, Characterization of tin selenides synthesized by high-energy milling, Acta Montan. Slov. 16 (2011) 123.

[42] Y. Cui, A. Abouimrane, C.J. Sun, Y. Ren, K. Amine, Li-Se battery: absence of lithium polyselenides in carbonate based electrolyte, Chem. Commun. 50 (2014) 5576.

[43] Y. Zou, Y. Wand, Sn@CNT nanostructures rooted in graphene with high and fast Li-storage capacities, ACS Nano 5 (2011) 8108. 
[44] J. Cai, Z. Li, P.K. Shen, Porous SnS nanorods/carbon hybrid materials as highly stable and high capacity anode for Li-ion batteries, ACS Appl. Mater. Interfaces 4 (2012) 4093.

[45] J. Qin, C. N. He, N. Q. Zhao, Z. Y. Wang, C. S. Shi, E. Z. Liu, J. J. Li, Graphene networks anchored with Sn@graphene as lithium ion battery anode, ACS Nano 8 (2014) 1728.

[46] S. Goriparti, E. Miele, F. De Angelis, E. Di Fabrizio, R. Proietti Zaccaria, C. Capiglia, Review on recent progress of nanostructured anode materials for Li-ion batteries, J. Power Sources 257 (2014) 421.

[47] L. Zeng, W. Zeng, Y. Jiang, X. Wei, W. Li, C. Yang, Y. Zhu, Y. Yu, A Flexible porous carbon nanofibers-selenium cathode with superior electrochemical performance for both Li-Se and Na-Se batteries, Adv. Energy Mater. 5 (2015) 1401377.

[48] C.P. Yang, Y.X. Yin, Y.G. Guo, Elemental selenium for electrochemical energy storage, J. Phys. Chem. Lett. 6 (2015) 256. 


\section{Ball milling}

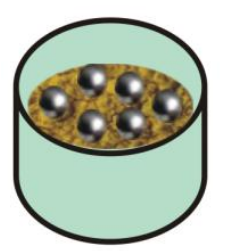

Nanosized $\mathrm{SnSe}$
2. Electrospinning

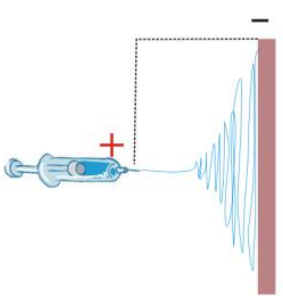

SnSe@PAN
3. Heating

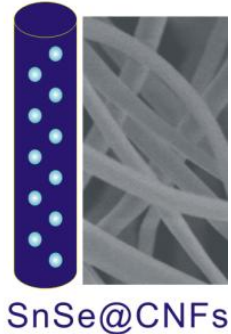

Scheme 1. Schematic representation of the synthesis process of SnSe/C hybrid nanofibers.

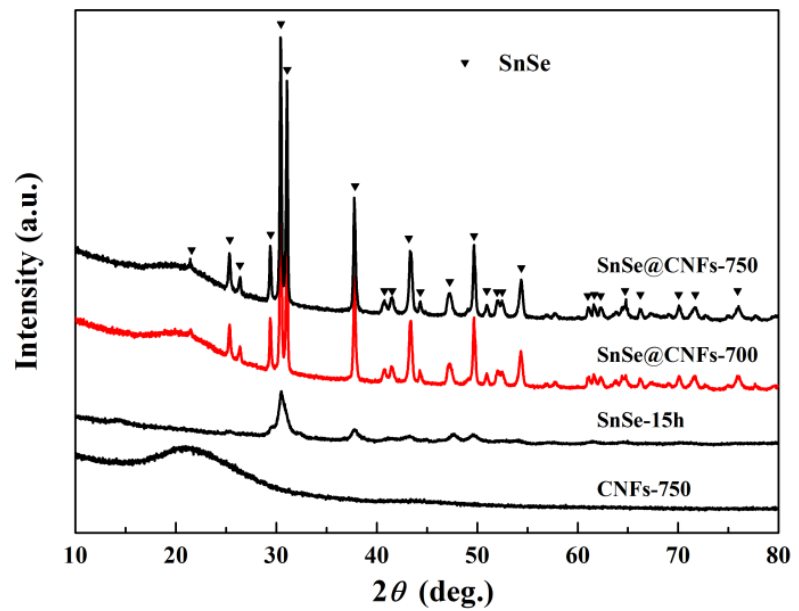

Fig. 1. XRD patterns of SnSe@CNFs-750, SnSe@CNFs-700, SnSe-15h, and CNFs-750. 


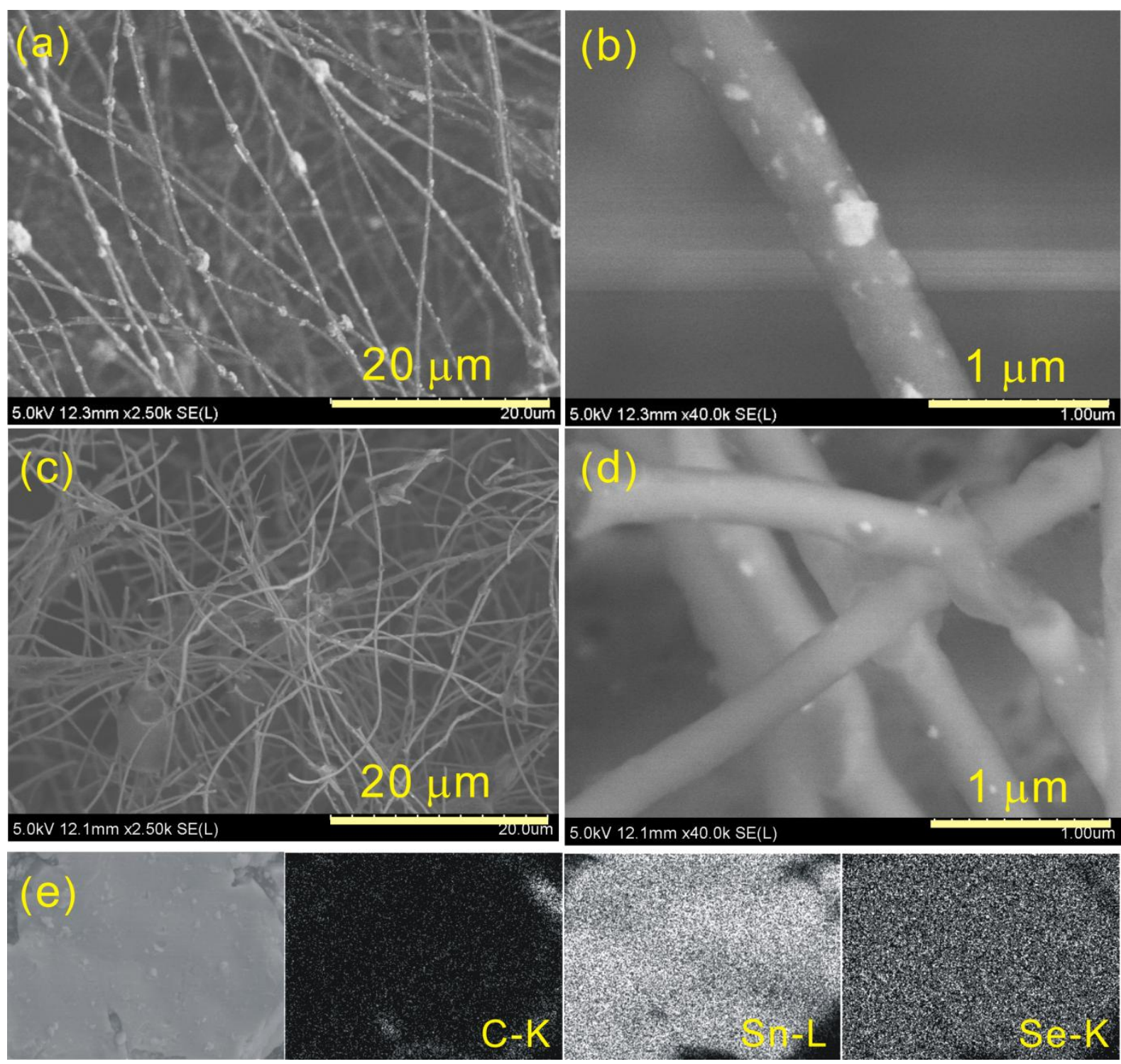

Fig. 2. SEM images of as-electrospun (a, b) and carbonized (c, d) SnSe@CNFs. (e) Elemental mapping images of C, Sn, and Se of EDS for a cold-pressed SnSe@CNFs-750 pellet. 

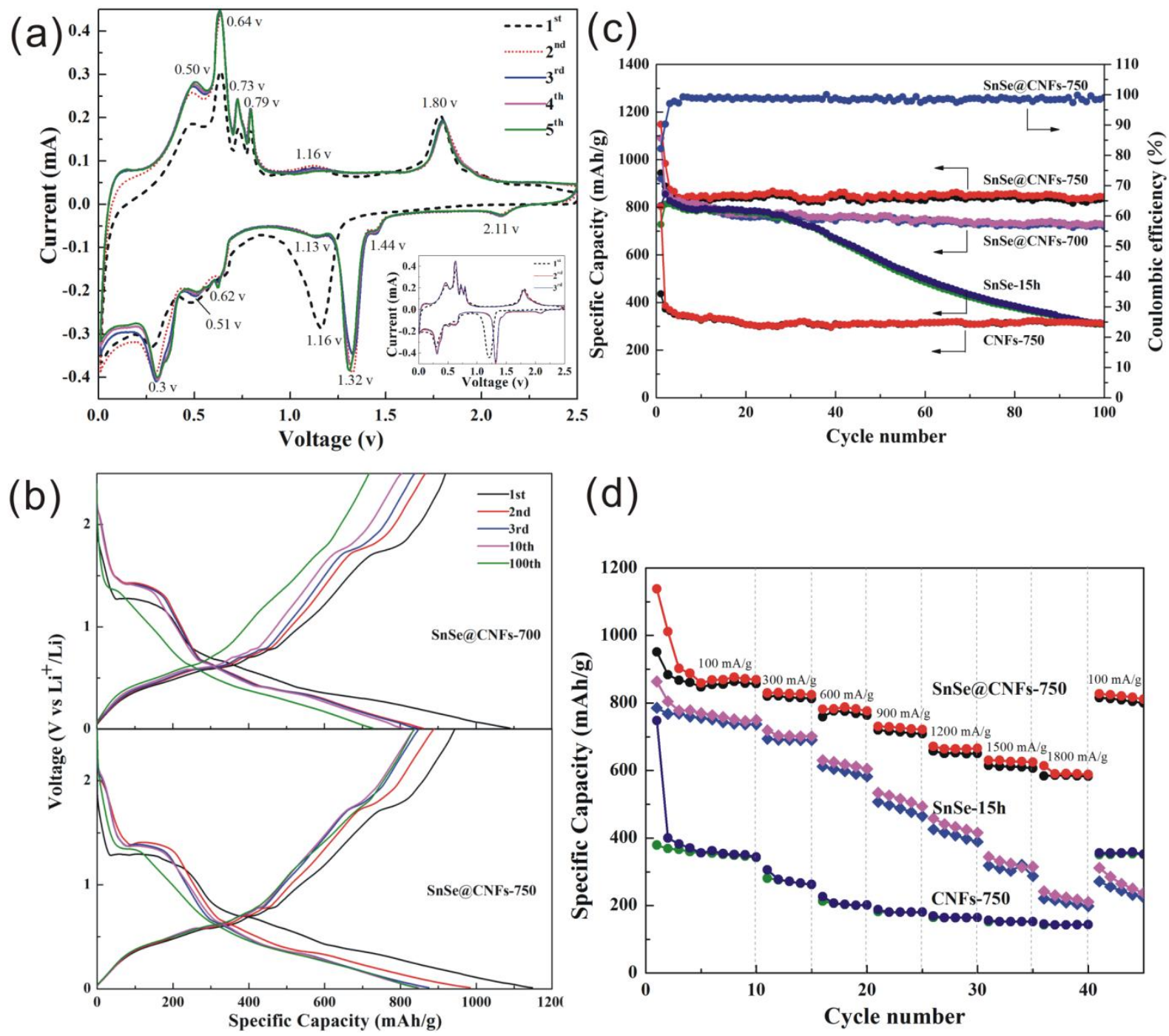

Fig. 3. (a) Cyclic voltammograms of SnSe@CNFs-750 at a scan rate of $0.1 \mathrm{mV} / \mathrm{s}$. Inset: Cyclic voltammograms of SnSe-15h. (b) Galvanostatic charge-discharge profiles of selected cycles for SnSe@CNFs-700 and SnSe@CNFs-750 at a current rate of 200 mA/g. (c) Cycling performance and Coulombic efficiency of various samples at a current density of $200 \mathrm{~mA} / \mathrm{g}$. (d) Rate capability of SnSe@CNFs-750, SnSe-15h, and CNFs-750.

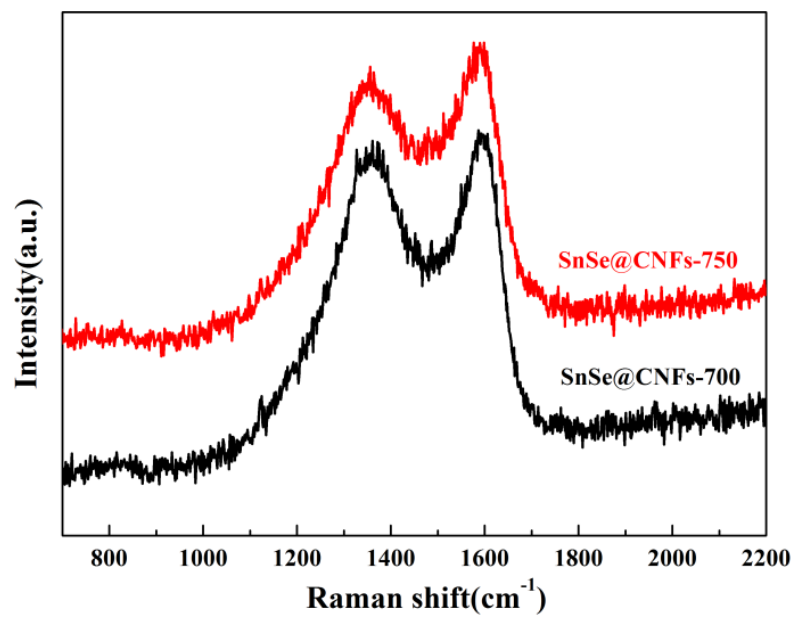

Fig. 4. Raman spectrum of SnSe@CNFs-700 and SnSe@CNFs-750. 

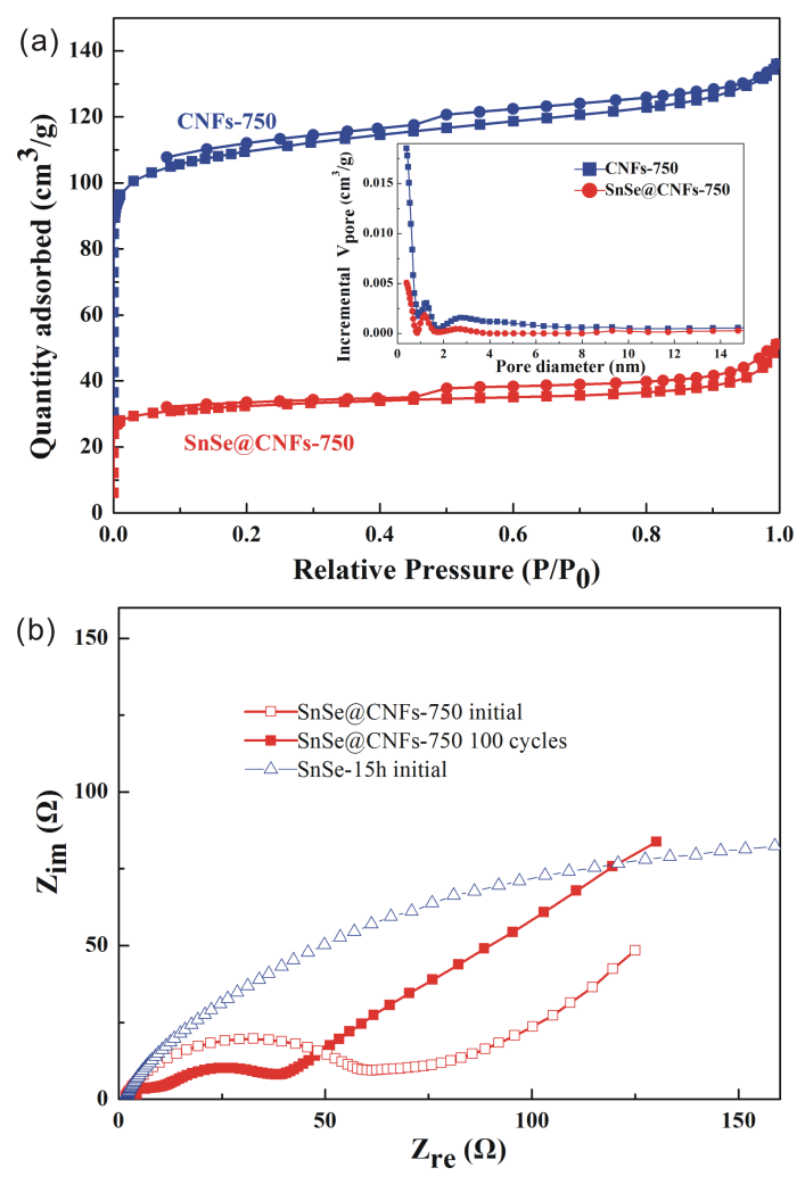

Fig. 5. (a) Nitrogen adsorption/desorption isotherm curves of CNFs and SnSe@CNFs. The inset shows the pore-size distribution. (b) Nyquist plots of SnSe@CNFs-750 and SnSe-15. 

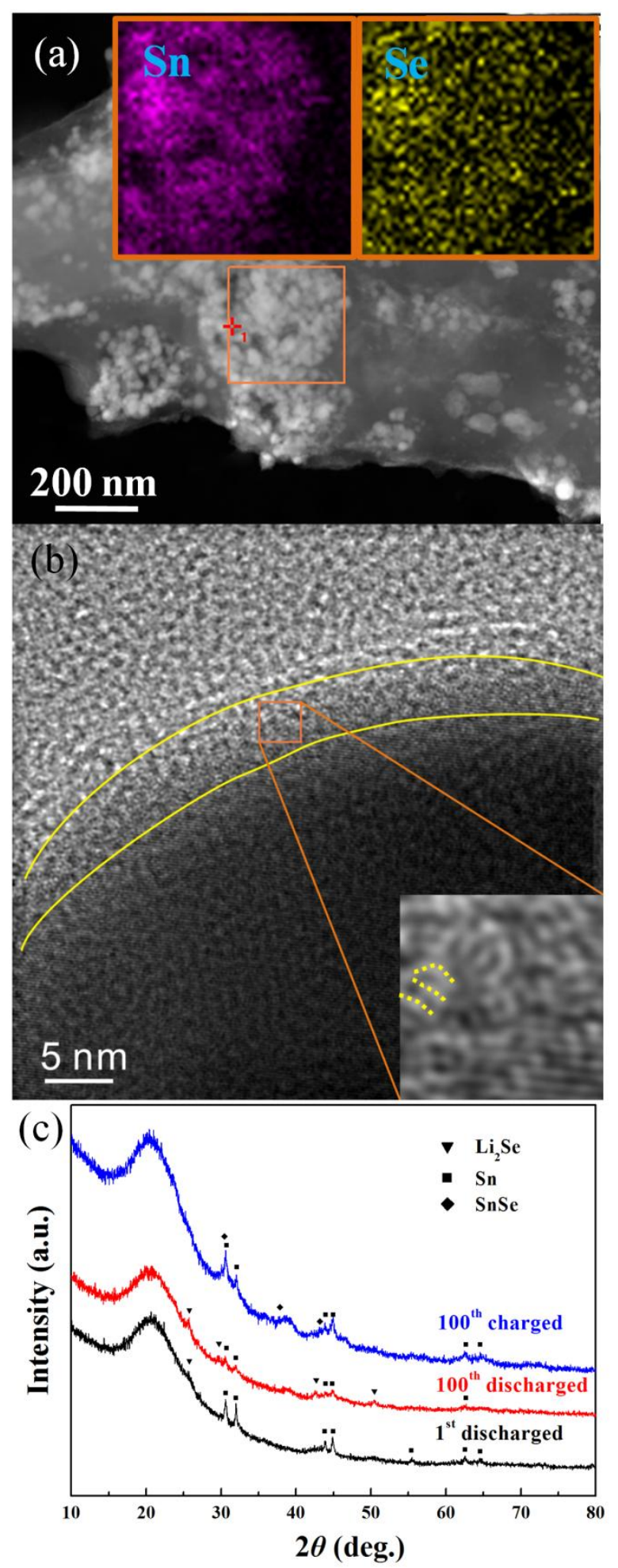

Fig. 6. Sn and Se Elemental mapping (a) and HR-TEM image (b) of SnSe@CNFs-750 after tenth discharging. (c) Ex situ XRD patterns of SnSe@CNFs-750 after fully discharging or charging. 